\title{
2D und 3D Kernspintomographie von 23NA im Gehirn
}

Scheidegger, M ; Sauer, M ; Bösiger, $\mathrm{P}$

DOI: https://doi.org/10.1515/bmte.1988.33.s2.155

Posted at the Zurich Open Repository and Archive, University of Zurich ZORA URL: https://doi.org/10.5167/uzh-155415

Journal Article

Published Version

Originally published at:

Scheidegger, M; Sauer, M; Bösiger, P (2009). 2D und 3D Kernspintomographie von 23NA im Gehirn. Biomedizinische Technik. Biomedical engineering, 33(Erg. Bd. 2):155-156.

DOI: https://doi.org/10.1515/bmte.1988.33.s2.155 


\title{
2D und 3D Kernspintomographie von ${ }^{23}$ NA im Gehirn.
}

\author{
M. Scheidegger, M. Sauer, P. Bösiger
}

\author{
Institut für Biomedizinische Technik und Medizinische Informatik der \\ Universität und ETH Zürich
}

In Virgäuzuug zu den Ergeb)nissen der konventionellen 111 hernspintomographie ist von Bildern, die auf den Signalen von ${ }^{23} \mathrm{Na}$ heruhen, komplementäre und vermehrt funktionelle Information 211 erwarten.

${ }^{23} \mathrm{~N}$ t lonen sind im gesunden (iehirn hauptsächlich im extrazellulären Jaum zu finden mit einer honzentration von etwa 150 mMol pro Liter. Die Natrium-halium lonenpumpe bewirkt, dass in den Zellen nur ca. $12 \ldots 15 \mathrm{mMlol} / \mathrm{l}$ Natrium-lonen vorhanden sind. Da aber der intrazellulïre Raum im Gehirn ca. $75 \%$ des Volumens ausfïll, beträgt die durchschnittliche Konzentration nur ca. $45 \mathrm{mMlol} / \mathrm{l}$.

Der Zusanmenbruch dieser lonenpumpe, wie z.B. in Falle eines llirninfarktes (Zelltod), kann zu lokalen Konzent rationszunahmen von bis zu $300 \%$ fïlirell. Auch die Ausdelinung des extrazellulären Raumes (Ödem) hat einen Anstieg der ${ }^{23} \mathrm{Na}$ lonenzahl zur Folge. Die transversalen Relaxationszeit en $T_{2}$ dieser verschiedenen (icwebranteile variieren stark [1] [2], was in Tal). 1 dargestellt ist. De-walb ändert die lokale durchschnittliche Relaxationszeit $T_{2}$ anch bei Ciewebeveränderungen. Von anderer Seite $[1]$ wird von einer horrelation $z$ wischen Malignität eines Tumors und intrazellulïrer Nat rium-honzent ration berichtet.

Es ist vielversprechend, die Möglichkeiten von ${ }^{23} \mathrm{Na} \mathrm{KST}$ abzustecken, im Ilinblick auf eine event uelle Frühdiagnose von Hirninfarkten im hontrast zu Hirnblutungen oder zur Unterscheidung 'Suntor - umgelendes Ödem. Dabei ist zu beachten, dass die Konzentration von ${ }^{23} \mathrm{Na}$ in Gewebe ca. $2000 \mathrm{mal}$ kleiner ist als diejenige von Protonen. Ausserdem sind die Relaxationszeit en kürzer, und die MK-Sensitivität beträgt, verglichen mit 11 , nur ca. $10 \%$. Berücksichtigt man alle diese Faktoren, so ergibt sich ein ungefähr 50 '000mal kleinerer Signal-Rauschabstand als bei Wasserstofnbildern.

\section{Natrium MR Abbildungsverfahren}

In der konventionellen III KST wird heute vieler Vorteile wegen fast ausschliesslich die Fouricr Ze ugmatographic als Abbildungsverfahren verwendet. Hingegen ist die Fourierabbildung für ${ }^{23} \mathrm{Na}$ wegen den kurzen Relaxationszeit en $T_{2}$ schlecht geeignet, da Phasentioliergradienten zwischen Anregung und Albtastung benötigt werilen.

W'icht igst es Erfordernis für eine höchstmögliche Signalausbeute ist, dass das Signal nach der Anregung so rasch als möglich abgetastet wird, damit möglichst wenig von den schenell zerfalleniden
Signalanteilen verlorengehen, die vermutlich die interessante Information beinhalten. Mögliche W'ge dazu sind folgende:'."

- keine l'hasenkodiergradienten zwischen Anregung und Abtastung;

- keine langen selektiven Anregungspulse.

Da beim Projektions-Rclionstruktionsverfahren, im folgenden ' $P R^{\prime}$ genannt, die Ortsinformation durch die wechselnden Richtungen des A uslesegradienten erhalten wird, fallt der bei der Fourieralbi,jdung gebrauchte Phasenkodiergradient weg. Die Signalabtastung kann früher beginnen.

Sowoll Fourier- wie $P R$-Abbildungen benötigen im $2 D$ Fall eine schichtselektive $A$ nregung mit ent sprechend langen selektiven nlf-Pulsen. Bei der $3 D$ Erweiterung des $P R$ Verfahrens hingegen kann clas gesamte Volumen mit einem kurzen, nichtselektiven Puls angeregl werden. Zusäızlich bieten $3 D$ Verfahren gegenüber $2 D$ Abbildungen mehr Schichten, das heisst melır Information, mit dem gleichen $S / R$ - $\Lambda$ bstand in der gleichen Messzeit. Deshall, ist die dreidimensionale Rekonstruktion von Projektionen die Methode der Wahl zur Ablsildung von ${ }^{23} \mathrm{Na}$ Kernen.

\section{1 $3 D P R$ Sequenz}

Fig. 1 zeigt die Sequenz von $P R 3 D$. Auf einen kurzen, nichtselektiven Anregungspuls erfolgt eine De- und sofort anschliessend eine Rephasierung der Spins in der Ausleserichtung, so dass diese am Anfang der Abtastperiode in Phase sind. Weitere Echos werden wahlweise durch Ciradient enumkehr oder durch 180" Pulse erzeugt. Der 'acquisition delay', die benötigte Zeit zwischen Anregungspuls und Beginn des Abtastens des freien Induktionszerfalles (FID), ist dalsei unalshängig von der Bildaufösung und nur durch die Hardware bestinumt (Schalten von (iradienten, coil ringing, eddy currents) und beträgt in unserem Fall üblicherweise 2.5 msek. Mit den gleichen Hardware-Einschränkungen beträgt diese Zeit ca. 6 msek für Fourierabhildungen mit einer Aufösung von 3.9 mum pro Bildpunkt und ca. 10 msek fïr eine Bildaufösung von $2 \mathrm{~mm}$.

Wenngleich das $3 D P R$ Verfahren optimal für ${ }^{23} \mathrm{Na} \mathrm{KST} \mathrm{im}$ llinblick auf $S / R$ und Anzahl gemessene Schichten pro Zeit ist, entstehen dadurch oft sehr lange Messzeiten (28 Minuten in unserem Fall) und lange Rekonstruktionszeiten, bedingt durch den komplexen Algorithmus. Für viele Fälle wäre eine $2 D P R$ Sequenz sehr nützlich, wenn sie den gleich kurzen 'acquisition delay' auf. weist wie das $3 D$ Gegenst ück.
(1), 


\begin{tabular}{|c|c|c|c|c|}
\hline Gewebe & $T_{2}$ echnell & $T_{2}$ langsam & $T_{2}$ mittel & $T_{1}$ \\
\hline Salzlösung & 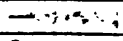 & $57-\cdots$ & $57 \ldots$ & 57 \\
\hline intrazellulär & $2 \ldots$ & $\ldots 16$ & 8 & $15 \ldots 25$ \\
\hline extrazellulär & - & $20 \ldots 40$ & $20 \ldots 40$ & $30 \ldots 50$ \\
\hline vaskulär & - & $30 \ldots 50$ & $30 \ldots 50$ & $40 \ldots 50$ \\
\hline
\end{tabular}

Tab. $1 .{ }^{23} \mathrm{Na}$ Relaxationszeiten im Gewebe

( [1] [2] und eigene Messungen; alle Zeiten in msek )

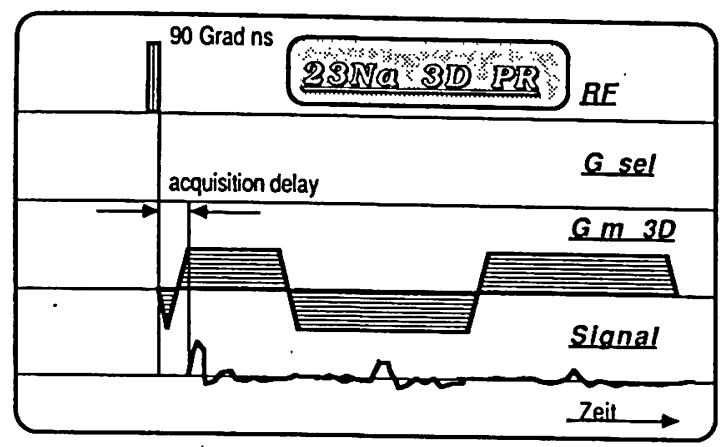

Fig. $1 .{ }^{23} \mathrm{Na} 3 D P R$ Sequenz

\subsection{Subtraktive Schichtselektion (2DPR)}

Fig. 2 zeigt die Sequenz für eine subtraktive Schichtselektion für 2DPR. Die Schichtselektion wird erreicht durch einen selektiven $180^{\circ}$ Inversionspuls und einen dazugehörigen Phasenzyklus. Da der folgende Anregungspuls nicht selektiv zu sein braucht, kann er sehr kurz gehalten werden und als Folge auch der 'acquisition delay'. Die Abtastung einer Gradientenrichtung erfordert mindestens 2 wei Einzelmessungen. In der ersten Messung wird der $180^{\circ}$ Puls weggelassen, und der FID enthält demzufolge Signalanteile aus dem ganzen Objekt. Während der zweiten Messung invertiert der $180^{\circ}$ Puls die Spins innerhalb der ausgewählten Schicht. Dieser zweite FID enthält zwei Signalanteile: eine Komponente mit Spins ausserhalb der Schicht mit der gleichen Phase wie in der ersten Messung und eine Komponente mit invertierter Phase von den Spins in der Schicht. Eine Subtraktion der zweiten Komponente von der ersten ergibt ein Signal mit nur Anteilen aus der gewählten Schicht.

Wegen Ungenauigkeiten von Hf-Pulsen muss in der Praxis der in Tab. 2 gezeigte Phasenzyklus über 4 Messungen verwendet werden, um störende Echos 2 wischen dem $90^{\circ}$ und dem $180^{\circ}$ Puls auszulöschen.

Anhand eines in Schichtrichtung ausgedehnten Phantorns wurden dabei verschiedene Pulsformen evaluiert. Die für selektive $90^{\circ}$ Pulse häufig gebrauchten 'sinc-Gauss' Pulse ergaben dabei (mit einer Simulation übereinstimmende) sehr schlechte Resultate: Schichtprofile mit ungenügenden Flankensteilheiten. Sehr gute Ergebnisse lieferten frequenzmodulierte Inversionspulse.

\section{Rekonstruktion der $P R 3 D$ Messdaten}

Nach dem Mitteln über eine Gradientenrichtung werden die Messdaten phasenkorrigiert. Eine $1 D$ Fouriertransformation ergibt Werte in Radon-Raum des Objekts (im 2D Fall entspricht dies einer Projektion). Un die Spindichte auf einem $3 D$ Gitter von $n^{3}$ Punkten $2 u$ rekonstruieren, muss der Auslesegradient in Cn.

\begin{tabular}{||l|l|l|l||}
\hline Messung & $180^{\circ}$ PILS & $90^{\circ}$ PIILS & EMPfängeh \\
\hline 1 & 0 & $\mathrm{X}+$ & + \\
\hline 2 & $\mathrm{X}+$ & $\mathrm{X}+$ & - \\
\hline 3 & 0 & $\mathrm{X}+$ & + \\
\hline 4 & $\mathrm{X}-$ & $\mathrm{X}+$ & - \\
\hline
\end{tabular}

Tab. 2. Phasenzyklus subtraktive Schichtselektion

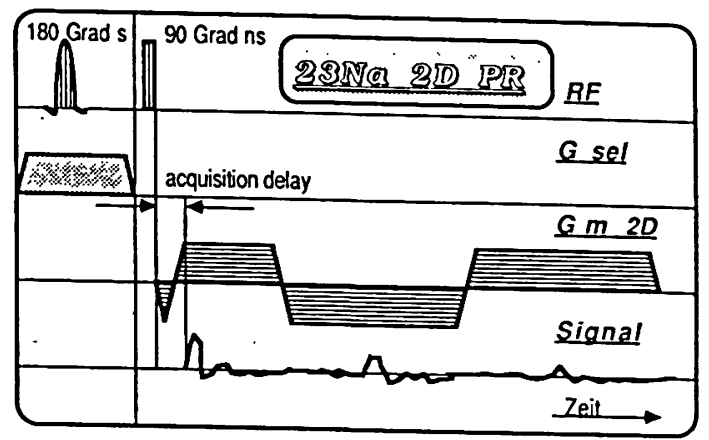

Fig. $2 .{ }^{23} \mathrm{Na} 2 D P R$ Sequenz

$n^{2}$ verschiedene Richtungen gelegt werden. Die Richtungen sind so gewählt, dass der ganze Raum möglichst homogen abgetastet wird.

Die Bildrekonstruktion erfolgt mittels eines diskreten Radon Inversionsalgorithnus (filt ered backprojection), und Schichten daraus werden zur Darstellung extrahiert. Die Rekonstruktionszeit beträgt für ein Echo und 14 Schichten bei einer Auflösung von 64 mal 64 Punkten ca. 1 Stunde auf einem Array Prozessor AP500.

\section{Experimente und Resultate}

Diese Sequenzen sind auf einem konunerziellen Philips 1.5 Tesla Ganzkörpergerät GYROSCAN S15 implementiert worden. Die wichtigsten Parameter sind: Schichtdicke $7.8 \mathrm{~nm}$, Bildmatrix 64 mal 64 Punkte; Bildauflösung 3.9 mm; Repetitionszeit der Messungen 65 msek. Die $3 D$ Aufnahmen, die 28 Minuten dauern, sind aus 22'000 Einzelmessungen rekonstruiert; $5 ` 00$ Ciradientenrich. tungen à je 4 Messungen. Die Echozeiten sind: $2.8 \mathrm{~ms}, 13 \mathrm{~ms}, 23$ ms.

Diese Parameter sind in ausgedehnten Phantom- und Prohandenstudien optimiert worden. Erste Bilder von Patienten mut bekannter Diagnose llirninfark: bestätigten den hohen Bildhontrast $z$ wischen Infarkt und umgebendem Gewebe. I'ntersuchungen an Patienten mit frischen Mirninfarkten, die wenige Stunden zurückliegen, laufen an.

\section{Literatur}

[1] Hilal et al; $6^{\text {th }}$ annual meeting SMRM 1987, NY p. 243

[2] Burstein D., Fossel E.; J. Magn. Reson. in Medicine 4, 261-273 (1987)

[ 3 ] Mckinnon G., Bösiger P.; Patent angemeldet (19si) 